\title{
Triiodothyronine supplementation and cytokines during cardiopulmonary bypass in infants and children
}

\author{
James R. Priest, MD, MA, ${ }^{\mathrm{a}, \mathrm{b}}$ April Slee, MS, ${ }^{\mathrm{c}}$ Aaron K. Olson, MD, ${ }^{\mathrm{a}}$ Dolena Ledee, $\mathrm{PhD},{ }^{\mathrm{a}}$ \\ Fionnuala Morrish, $\mathrm{PhD},{ }^{\mathrm{d}}$ and Michael A. Portman, $\mathrm{MD}^{\mathrm{a}}$
}

\begin{abstract}
Objective: The Triiodothyronine Supplementation in Infants and Children Undergoing Cardiopulmonary Bypass (TRICC) study demonstrated a shortened time to extubation in children younger than 5 months old undergoing cardiopulmonary bypass for congenital heart surgery with triiodothyronine supplementation. Cardiopulmonary bypass precipitates a systemic inflammatory response that affects recovery, and triiodothyronine is related to cytokine mediators of inflammation. We sought to investigate the preoperative cytokine levels by age and relationship to the triiodothyronine levels and to examine the effect of the cytokine levels on the time to extubation.
\end{abstract}

Methods: We measured 6 cytokines at preoperative time 0 and 6 and 24 hours after crossclamp removal in 76 subjects.

Results: The preoperative cytokine levels were related to both the triiodothyronine levels and the patient age. The postoperative cytokine levels were predictive of the triiodothyronine levels at $6,12,24$, and 72 hours. Preoperative CCL4 was associated with an increased chance of early extubation. Inclusion of the cytokines did not change the relationship of triiodothyronine to the time to extubation, and the postoperative course of interleukin- 6 was independently associated with a decreased chance of early extubation.

Conclusions: The preoperative and postoperative cytokine levels, in particular, interleukin- $1 \beta$, showed complex time-dependent relationships with triiodothyronine. The data suggest that cytokine-mediated suppression of triiodothyronine plays an important role in determining the clinical outcome after cardiopulmonary bypass. (J Thorac Cardiovasc Surg 2012;144:938-43)

Supplemental material is available online.

Since the development of cardiopulmonary bypass (CPB) in the 1950s, its use has been associated with an inflammatory response, although early reports attributed the pathologic and radiologic findings of lung injury to anoxia or inadequate perfusion. ${ }^{1}$ Later investigations associated CPB with complement activation, immunoglobulin deficiency, and an impaired mitogen response, suggesting a broad

From the Seattle Children's Hospital ${ }^{\mathrm{a}}$ and University of Washington, Seattle, Wash; Division of Pediatric Cardiology, ${ }^{\mathrm{b}}$ Stanford University School of Medicine and Lucile Packard Children's Hospital, Palo Alto, Calif; Axio Research, ${ }^{\text {c }}$ Seattle, Wash; and Fred Hutchinson Cancer Research Center, ${ }^{\mathrm{d}}$ Seattle, Wash.

This research was supported by a grant to M.A. Portman from the U.S. Food and Drug Administration Orphan Product Development Program (grant no. BR01 FDR-1971-01) and the University of Washington Clinical and Translational Science Award (grant no. 1ULI RR025014-01) from the National Center for Research Resources, a component of the National Institutes of Health. The drug was supplied by King Pharmaceutical.

Disclosures: Authors have nothing to disclose with regard to commercial support.

Received for publication Nov 18, 2011; revisions received April 16, 2012; accepted for publication May 17, 2012; available ahead of print June 28, 2012.

Address for reprints: Michael A. Portman, MD, Seattle Children's Research Institute, 1900 9th Avenue, Seattle, WA 98101 (E-mail: michael.portman@seattlechildrens. org).

0022-5223/ $\$ 36.00$

Copyright (c) 2012 by The American Association for Thoracic Surgery doi:10.1016/j.jtcvs.2012.05.063 immunologic perturbation. ${ }^{2,3}$ Contemporary research has demonstrated a clear CPB-related inflammatory response, induced by the synthetic milieu of the bypass apparatus, with a presumed clinical effect on the postoperative outcomes in pediatric and adult populations. ${ }^{2-4}$ Research into ameliorating this CPB-related inflammatory response has spanned the disciplines of anesthesia, perfusion, surgery, pharmacology, and critical care..$^{5-9}$

Recently we conducted the Triiodothyronine (T3) Supplementation in Infants and Children Undergoing Cardiopulmonary Bypass (TRICC) study in a pediatric population requiring congenital heart surgery. ${ }^{10}$ The TRICC study concluded that $\mathrm{T} 3$ supplementation shortened the time to extubation (TTE) and improved cardiac function in children younger than 5 months old. A systematic review of T3 supplementation in the postoperative care of adults undergoing a variety of surgeries concluded that $\mathrm{T} 3$ improves the postoperative cardiac index. ${ }^{11}$ In adults with dilated cardiomyopathy, T3 supplementation improved the neuroendocrine hormone levels and measures of cardiac output. ${ }^{12}$

Some published data have suggested that cytokines might interact with the thyroid hormone axis. In the myocardium, evidence has shown that T3 directly modulates transcriptional regulation, histone modification, ion channels, and intracellular cations. ${ }^{13-15}$ Additional observational studies have suggested an effect of T3 outside the myocardium, reporting 


\section{Abbreviations and Acronyms \\ ANCOVA $=$ analysis of covariance \\ $\mathrm{CI}=$ confidence interval \\ $\mathrm{CPB}=$ cardiopulmonary bypass \\ IL = interleukin \\ MCP1 = monocyte chemotactic protein-1 \\ $\mathrm{T} 3=$ triiodothyronine \\ TRICC = Triiodothyronine Supplementation in \\ Infants and Children Undergoing \\ Cardiopulmonary Bypass \\ TTE $=$ time to extubation}

an inverse relationship between the pro-inflammatory cytokine interleukin (IL)-6 and T3 levels in the setting of CPB and infectious diseases in pediatric populations. ${ }^{16,17}$ Infusion of recombinant IL-6 into healthy adults has been shown to decrease T3 levels at 24 hours after infusion. ${ }^{18}$

We sought to explore the relationship of age and T3 supplementation to cytokine levels and outcomes in a subset of the TRICC cohort. The primary objectives of the present study were to investigate the preoperative cytokine levels by age and relationship to the T3 levels and to examine the pre- and postoperative cytokine levels for their effect on the TTE.

\section{METHODS \\ Study Population}

The study population has been previously described in detail in the TRICC report. ${ }^{10}$ In brief, of the 193 participants younger than 2 years old undergoing CPB for congenital heart surgery, we selected a subset for cytokine analysis. Patients were previously categorized as being at high or low risk according to an Aristotle score cutoff of 10, and the study included patients with a hypoplastic left heart. After enrollment, we randomized the participants to treatment with $\mathrm{T} 3$ or placebo using a complex stratification algorithm that was block randomized by surgical diagnosis. Also, patients received routine postoperative care by critical care physicians who were unaware of the treatment assignment. Steroid use varied according to the standard clinical protocols in use at the different institutions. We summarized the baseline characteristics of the cytokine subset alone by treatment status. Of the 193 participants in the TRICC cohort, we included the first 65 participants enrolled as a subset for cytokine analysis and an additional 11 with available serum to balance the risk and demographics. The institutional review boards at all participating centers approved the present study, and the parents of all participants provided written informed consent.

\section{Laboratory Testing}

Blood was sampled at 3 points: preoperative time 0 (before administration of T3) and 6 and 24 hours after crossclamp removal. Sampling involved multiplex measurement of 6 cytokines in a commercially available kit (IL$1 \beta$, IL-6, IL-8, IL-10, CCL4, and monocyte chemotactic protein-1 [MCP1], which bind to 4 different receptor classes involved in vascular inflammation).$^{19}$ Frozen human serum at $-80^{\circ} \mathrm{C}$ was thawed on ice and tested to assess the levels of 6 different cytokines. The cytokines were measured using the Bio-Plex Pro ${ }^{\mathrm{TM}}$ Human Cytokine Assay bead kit (Bio-Rad Laboratories, Hercules, Calif). The beads were added to wells with serum samples and incubated for 30 minutes. After incubation, the plates were washed and detection antibodies were added. After additional incubations and washes, the plates were run immediately on a Luminex $200^{\mathrm{TM}}$ System (Luminex Corporation, Austin, Tex) with data acquisition and MasterPlex analysis software (Hitachi Solutions America, South San Francisco, Calif).

\section{Statistical Analysis}

We used the $\mathrm{R}$ language and environment for statistical computing, version 2.8.1 (R Foundation for Statistical Computing, Vienna, Austria) and SAS (SAS Institute, Cary, NC) for all statistical analysis. Graphs were produced using Splus, version 6.2 (Tibco Software, Palo Alto, Calif). We did not perform correction for tests of multiple hypotheses. Patients who died or experienced an otherwise catastrophic outcome were excluded. For all analyses involving cytokine levels or T3, we transformed the raw values using the natural logarithm of the raw value +1 . Unless otherwise stated, the $\mathrm{T} 3$ and cytokine values were analyzed on the transformed scale to account for heavily skewed distributions to maintain a constant variance for regression modeling and statistical tests. We tested the relationships between age and risk using a Wilcoxon rank sum test and investigated the association between age and cytokine levels with linear least squares regression at baseline time 0 for each cytokine. As suggested by previous investigators, we also analyzed the IL-10/IL- 6 ratio in our analyses of age. ${ }^{20}$

We examined the preoperative relationship of $\mathrm{T} 3$ and cytokines with an analysis of covariance (ANCOVA) model that included all cytokines and the Aristotle risk class to account for the randomization stratification. High risk was determined by an Aristotle score greater than 10 . The postoperative ANCOVA models included the cytokine levels at hour 6 and risk as predictor variables for T3 levels at 6,12, 24, and 72 hours. To determine the natural relationship of the preoperative cytokine levels to the TTE, we included only the placebo patients in a Cox proportional hazards model. The outcome was extubation, and patients were censored at death or 7 days after intubation. To analyze the relationship of the cytokine response to TTE, we constructed a Cox proportional hazards model that included the time-dependent cytokine levels and time-dependent T3 levels to account for treatment. As a sensitivity analysis, the treatment assignment was added to this model. The covariates for ANCOVA and Cox proportional hazards models were chosen using stepwise selection to select the most important predictors for each outcome variable.

\section{RESULTS}

\section{Patient Characteristics}

Analysis of the patient demographics of the 76 participants within the cytokine subset and the remainder of the TRICC cohort was performed and identified the cytokine subset as younger, with greater risk and with elevated baseline T3 levels (Table E1). Within the cytokine subset, no statistically significant differences were found in the baseline characteristics when analyzed by treatment status. However, 5 more high-risk patients were in the treated group of the cytokine subset (Table 1). Risk was inversely related to age (Wilcoxon rank sum test, $P<.001$ ). Figure 1 displays a graph of the mean values and standard errors for the $6 \mathrm{cy}$ tokines over time in the placebo group. IL-6, IL-8, and IL10 were significantly increased after CPB at both 6 and 24 hours in the placebo group (Figure 1 and Table E2).

\section{Cytokines and Age}

The baseline levels of IL-6 $(-1.11 \mathrm{pg} / \mathrm{dL}$ for each month of age; $95 \%$ confidence interval $[\mathrm{CI}],-0.84$ to -1.03 ; $P=.005), \mathrm{IL}-8(-1.06 \mathrm{pg} / \mathrm{dL}$ for each month of age; $95 \%$ 
TABLE 1. Baseline characteristics of analyzed cytokine subset stratified by treatment status

\begin{tabular}{lccr}
\hline \multicolumn{1}{c}{ Characteristic } & $\begin{array}{c}\text { Placebo } \\
(\mathbf{n = 3 7 )}\end{array}$ & $\begin{array}{c}\text { Triostat } \\
(\mathbf{n = 3 9 )}\end{array}$ & $\begin{array}{r}\boldsymbol{P} \\
\text { value }\end{array}$ \\
\hline Male gender (n) & 15 & 15 & .96 \\
Risk stratification (n) & & & .45 \\
$\quad$ High & 13 & 18 & \\
$\quad$ Low & 24 & 21 & \\
Age (mo) & & & .69 \\
$\quad$ Median & 4.1 & 3.4 & \\
$\quad$ Range & $0-23.2$ & $0-19.1$ & \\
Baseline triiodothyronine (U) & 172 & 188 & .19 \\
Mean intubation time (h) & 94 & 113 & .67 \\
Mean crossclamp time (min) & 62 & 61 & .96 \\
Mean bypass time (min) & 97 & 97 & 1.00 \\
Deaths (n) & 1 & 3 & .64 \\
\hline All $P$ var
\end{tabular}

All $P$ values presented were derived from Student $t$ test, except for gender, risk stratification, and death, which were derived from a simple chi-square test.

$\mathrm{CI},-1.12$ to $-1.01 ; P=.033)$, and CCL4 $(-1.06 \mathrm{pg} / \mathrm{dL}$ for each month of age; $95 \% \mathrm{CI},-1.10$ to $-1.03 ; P=.001$ ) showed inverse relationships with months of age and IL$10(1.05 \mathrm{pg} / \mathrm{dL}$ for each month of age; $95 \% \mathrm{CI}, 1.01$ $1.47 ; P=.05)$ showed a trend toward a direct relationship with age (Figure 2). At baseline (time 0 ), IL-1 $\beta$ and MCP1 did not show statistically significant relationships with age; however, the IL-10/IL-6 ratio was directly related to age $(1.06 \mathrm{U} /$ month of age; $95 \% \mathrm{CI}, 1.03-1.08 ; P<.001)$.

\section{Cytokines and T3}

Because of the preprocedure homogeneity of the cohort, we used all patients in an ANCOVA model to determine the relationship between baseline risk and cytokine levels and baseline T3 (Figure 3). We found several measures to be inversely related to the baseline $\mathrm{T} 3$ level, including high-risk status $(-1.46 \log \mathrm{T} 3 \mathrm{ng} / \mathrm{dL}$ for high-risk status; $95 \% \mathrm{CI}$, -1.70 to $-1.25 ; P<.001)$, IL-6 $(-1.08 \log$ T3 ng/dL per ng/dL of IL-6; $95 \%$ CI, -1.16 to $-1.01 ; P \leq .001)$, and IL-1 $\beta(-1.45 \log \mathrm{T} 3 \mathrm{ng} / \mathrm{dL}$ per ng/dL of IL- $1 \beta ; 95 \% \mathrm{CI}$, -1.82 to $-1.16 ; P=.002)$. In addition, we found a direct relationship between IL-8 and baseline T3 (1.11 log T3 ng/dL per ng/dL of IL-8; 95\% CI, 1.03-1.19; $P=.004)$.

We used ANCOVA to examine the relationships between cytokines and T3. In the placebo patients, we modeled all cytokines at 6 hours after crossclamp removal (the earliest postoperative measure) versus the T3 levels at 6 hours and determined that risk status, IL- $1 \beta$, and IL- 10 were significantly associated with the T3 levels. Only IL- $1 \beta$ at 6 hours was inversely associated with the T3 levels at all points through 72 hours. IL-10 at 6 hours was inversely associated with the T3 levels at 12 and 24 hours, and high-risk status was inversely associated with the T3 levels at 6 hours (Figure 4 and Table E3).

\section{Cytokines and Clinical Parameters}

Preoperative IL-8 was associated with a decreased chance of early extubation (hazard ratio, $0.21 ; 95 \% \mathrm{CI}$, $0.06-0.78 ; P=.02$ ), and preoperative CCL4 was associated with an increased chance of early extubation (hazard ratio, $1.51 ; 95 \%$ CI, 1.11-2.06; $P=.001$; Figure E1).

Among all patients, modeling T3, IL-6, and CCL4 as time-dependent covariates, greater IL-6 levels were associated with a reduced chance of early extubation (hazard ratio, $0.70 ; 95 \%$ CI, 0.57-0.85; $P<.001$ ), as was the baseline high-risk status (hazard ratio, 0.47; 95\% CI, $0.26-0.85 ; P=.01)$. High T3 levels were associated with an increased chance of early extubation (hazard ratio, 1.43 ; 95\% CI, 1.25-1.63; $P<.001$ ), as were high CCL4 levels (hazard ratio, 1.34; 95\% CI, 0.97-1.85; $P=.08$;
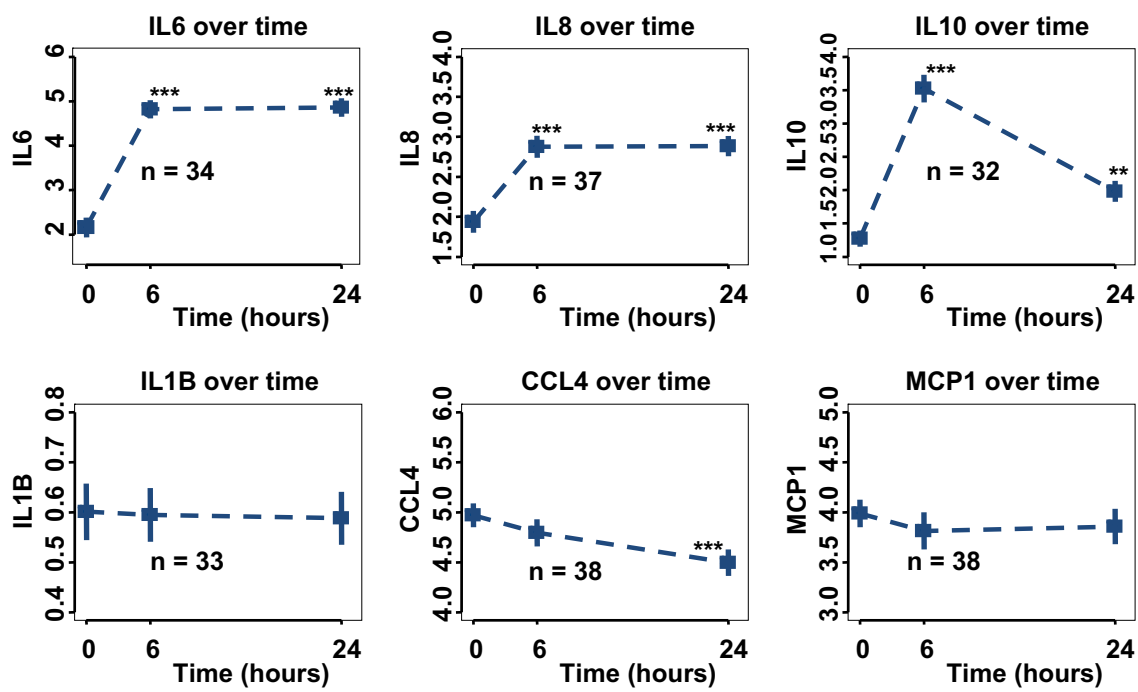

FIGURE 1. Cytokine levels during time course of the trial in placebo patients $(\mathrm{n}=37)$. $* * P<.01$; $* * * P<.001$. IL, Interleukin; $C C L 4$, chemokine (C-C motif) ligand 4; MCPI, monocyte chemotactic protein-1. 

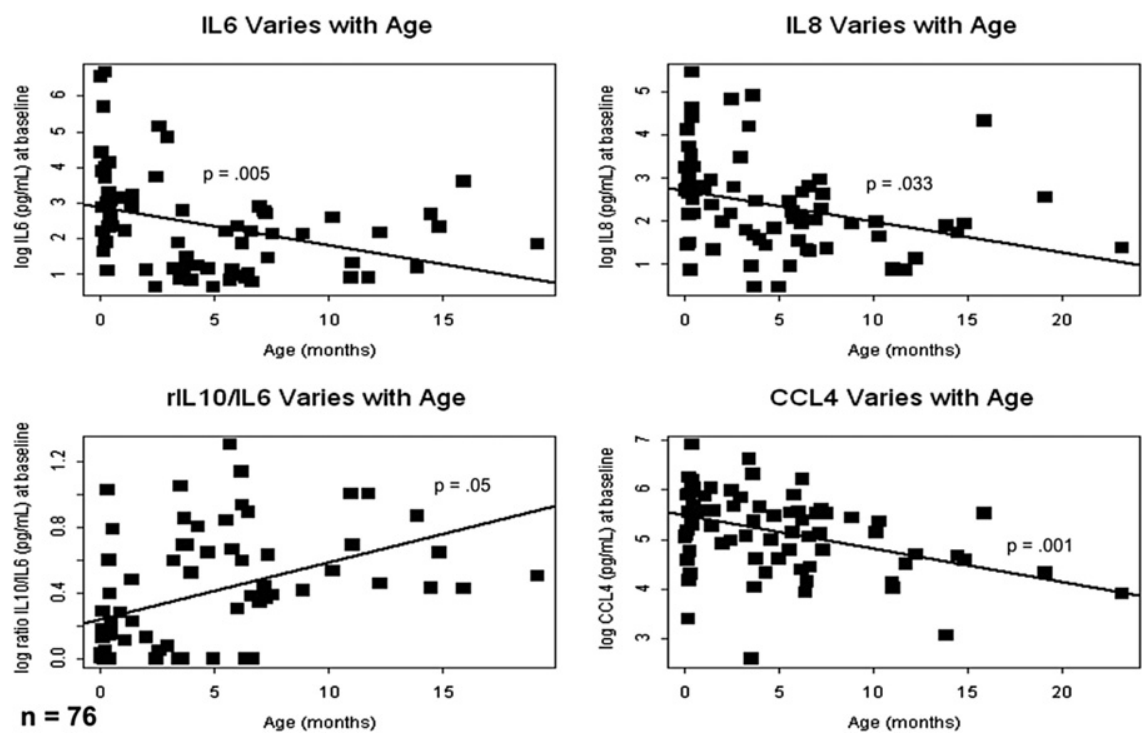

FIGURE 2. Preoperative interleukin-6, interleukin-8, and CCL4 levels were inversely related to patient age (n = 76). IL, Interleukin; $C C L 4$, chemokine (C-C motif) ligand 4.

Figure 5). These results were similar when randomization assignment was added to the model.

\section{DISCUSSION}

The major objective of the present study was to define the relationships among T3, cytokine levels, and the clinical outcomes in the study population of children undergoing CPB. As would be expected from our previously reported trial data, the T3 level throughout the early postoperative period is a powerful predictor of the TTE. We observed complex pre- and postoperative relationships among the measured cytokines, thyroid hormone levels, and the TTE.

In our analysis of cytokines and age, younger patients had greater levels of the pro-inflammatory cytokines IL $1 \beta$, IL-6, and IL8 and a lower level of the anti-inflammatory IL-10/ IL-6 ratio than the older patients (Figure 2). In aggregate, these finding might imply that younger patients undergoing

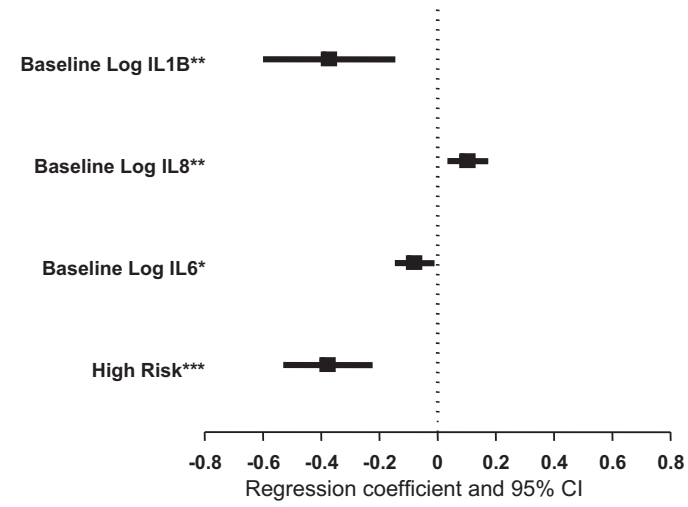

FIGURE 3. Forest plot of $\beta$-coefficients from linear regression analysis of baseline cytokine levels and baseline triiodothyronine levels $(\mathrm{n}=37) . * P<$ $.05 ; * * P<.01 ; * * * P<.001$. IL, Interleukin; $C I$, confidence interval. heart surgery have a pro-inflammatory cytokine profile that diminishes with age. As expected, risk and age were also inversely related. Little is known about the steady-state cytokine levels of healthy or ill neonates, and our small cohort and study design were not suited to evaluate whether the pro-inflammatory cytokine profile of the younger patients was independent from their greater preoperative risk or congenital cardiac lesion.

Although we did not observe a relationship between the preoperative T3 level and TTE, we did note that the 2 preoperative cytokines related to TTE-IL-8 and CCL4-both bind chemokine receptors. The chemokines and their receptors are known to regulate leukocyte trafficking and migration in the setting of myocardial inflammation, which might suggest a process by which the cytokine levels influence myocardial function in the pre- and postoperative periods. ${ }^{21}$

Additionally, we found relationships among IL- $1 \beta$, IL-8, and $\mathrm{T} 3$ at baseline. This finding highlights the complex relationship between cytokines and the endogenous levels of $\mathrm{T} 3$ that was the very subject of our study intervention. The inverse relationship of IL- $1 \beta$ to the preoperative T3 level is of particular interest given the observation that IL- $1 \beta$ suppresses the secretion of thyroid-stimulating hormone from cultured rat anterior pituitary cells. ${ }^{22}$ Allan and colleagues $^{23}$ did not detect significant levels of IL- $1 \beta$ pre- or postoperatively in another population of young infants and children undergoing CPB. This contrasting finding might reflect differences in the study methods such as their use of a protein microarray chip versus our multiplex method or might have been due to experimental error. ${ }^{23}$ The preoperative findings might thus serve to illustrate the steady-state relationship between cytokines and the thyroid hormone axis, which is then altered by administration of T3. 

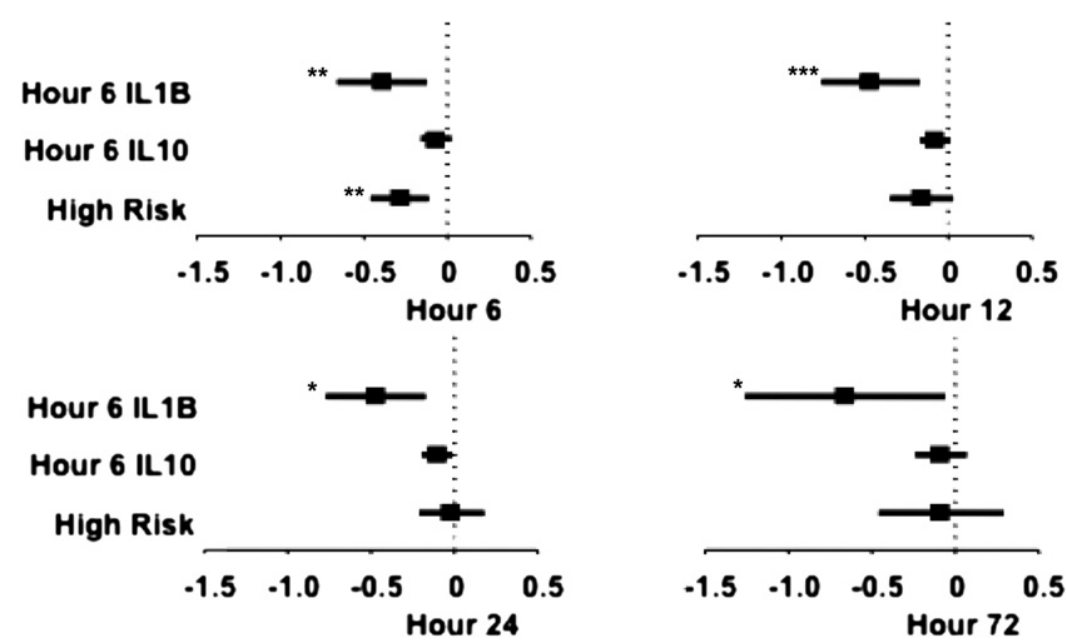

FIGURE 4. Forest plot of $\beta$-coefficients from linear regression analysis of 6-hour cytokine levels and triiodothyronine levels at 6, 12, 24, and 72 hours postoperatively $(\mathrm{n}=37)$. $* P<.05 ; * * P<.01 ; * * * P<.001 . I L$, Interleukin.

Consistent with the preoperative observation of an inverse relationship between IL-1 $\beta$ and T3 and the experimental data noted, T3 exhibited sensitivity to the IL-1 $\beta$ levels throughout the first 72 hours of the postoperative period (Figure 4). Considering the pre- and postoperative data together suggest that the improved TTE observed in patients treated with $\mathrm{T} 3$ results from the repletion of the T3 suppressed in patients with elevated IL- $1 \beta$. As a timedependent covariate, IL-6 was predictive of prolonged extubation. The Cox proportional hazard model that included the postoperative T3 level as a continuous variable instead of treatment status suggested that the serum levels of T3 captures most of the effect of treatment.

Anecdotally, clinicians have used T3 postoperatively for decades in patients slower to recover from CPB than expected or who display signs of low cardiac output

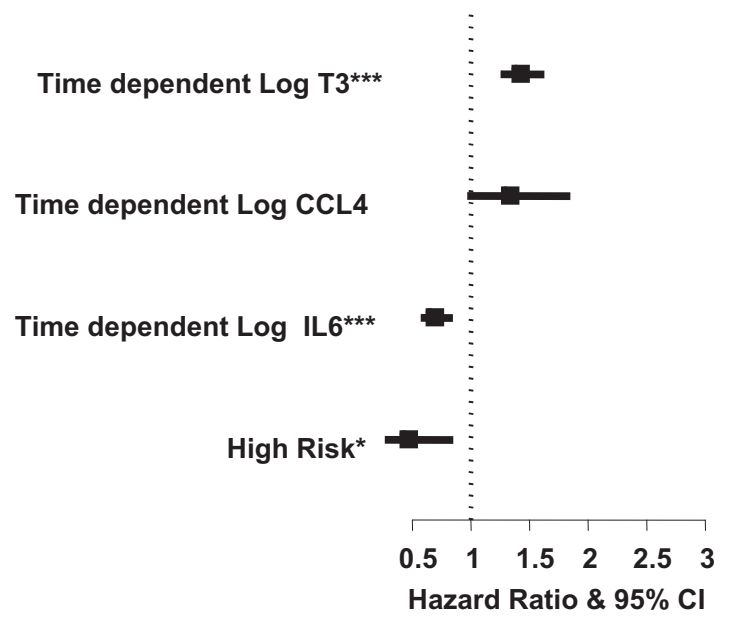

FIGURE 5. Forest plot of hazard ratio for time to extubation of timedependent cytokine levels, time-dependent triiodothyronine level, and risk $(\mathrm{n}=76) . * P<.05 ; * * * P<.001 . \mathrm{T} 3$ is scaled by $5 . I L$, Interleukin; CCLA, chemokine (C-C motif) ligand 4 ; $C I$, confidence interval. syndrome. Our data suggest that preoperative screening of certain cytokines, such as IL-6, IL-8, or CCL4, could help in the risk stratification of patients at risk of prolonged intubation. With regard to T3 treatment, using pre- and postoperative IL- $1 \beta$ levels might add predictive value to determining which patients could benefit from postoperative T3 supplementation.

The choice of cytokines was determined by the multiplex chips commercially available at the initial study. The chosen platform of assayed cytokines included both IL-6 and IL-10, which were reasonable candidates for the study according to the available data from previous studies. However, an exhaustive list of cytokines and their various isoforms is significantly larger than the 6 cytokines used in our study; thus, our data could not completely capture the nuanced and complex inflammatory response after CBP and the effect of T3 supplementation. Compared with the overall TRICC cohort, the cytokine subset was younger, at higher risk, and had elevated baseline T3 levels. Although we attempted to account for the difference between the TRICC cohort and the cytokine subset with multivariate analysis, such techniques can introduce the possibility of error, which we could not completely exclude. Finally, the cytokine relationships described were based on 76 patients with varying ages and diagnoses (Table E4); therefore, a larger trial is necessary to determine the broad applicability of the response of cytokines to CPB and T3 supplementation, particularly for children younger than 5 months old.

\section{CONCLUSIONS}

These data reinforce the important association between T3 and the clinical outcome, TTE, in young patients undergoing CPB. Complex relationships exist between and among the multiple cytokines, in particular IL- $1 \beta$, that vary with time during the postoperative course. Overall, 
these relationships suggest that $\mathrm{T} 3$ suppression is influenced by the cytokine response to both the underlying disease state and to CPB and that the effect of inflammation on the TTE can be corrected by T3 supplementation.

We thank the following participants from the original TRICC study: Seattle Children's Hospital-G. Cohen, L. Permut, M. Lewin, A. Cesan, M. B. Lee, C. Fearneyhough, K. Rodriquez, K. Gama, and E. Mano; University of California San Francisco-T. Karl, T. A. Tacy, and A. Azakie; Oakland Children's HospitalH. Patel, O. Reinhartz, and J. Simon; Children's Hospital of Los Angeles-L. Hastings and G. Kung; Sutter Medical Center-R. Mainwaring and T. Donnel; Texas Children's Hospital-A. Mott and R. Pignatelli; North Shore University Hospital-S. Danzi and I. Klein; Data Monitoring Committee, University of Washington-R. Knopp and F. Kim; Seattle Children's Hospital—B. Hardy and M. Cohen; and the independent medical monitor, Mayo Clinic-H. Burkhardt.

\section{References}

1. Kolff WJ, Effler DB, Groves LK. A review of four dreaded complications of open-heart operations: causes, avoidance, and treatment of acidosis, overoxygenation, heart-block, and pulmonary damage. BMJ. 1960;1:1149-53.

2. Fung M, Loubser PG, Undar A, Mueller M, Sun C, Sun WN, et al. Inhibition of complement, neutrophil, and platelet activation by an anti-factor D monoclonal antibody in simulated cardiopulmonary bypass circuits. J Thorac Cardiovasc Surg. 2001;122:113-22.

3. Stocker CF, Shekerdemian LS, Visvanathan K, Skinner N, Brizard CP, Carlin JB, et al. Cardiopulmonary bypass elicits a prominent innate immune response in children with congenital heart disease. J Thorac Cardiovasc Surg. 2004;127:1523-5.

4. Kuss O, von Salviati B, Borgermann J. Off-pump versus on-pump coronary artery bypass grafting: a systematic review and meta-analysis of propensity score analyses. J Thorac Cardiovasc Surg. 2010;140:829-35, e1-13.

5. Miller A, Lu CK, Wang S, Umstead TM, Freeman WM, Vrana K, et al. Pediatric cardiopulmonary bypass circuits: a review of studies conducted at the Penn State Pediatric Cardiac Research Laboratories. J Extra Corpor Technol. 2009;41:P50-8.

6. Nilsson L, Nilsson U, Venge P, Johansson O, Tyden H, Aberg T, et al. Inflammatory system activation during cardiopulmonary bypass as an indicator of biocompatibility: a randomized comparison of bubble and membrane oxygenators. Scand J Thorac Cardiovasc Surg. 1990;24:53-8.

7. Robertson-Malt S, Afrane B, El Barbary M. Prophylactic steroids for pediatric open heart surgery. Cochrane Database Syst Rev. 2007;4:CD005550.

8. Salvin JW, Scheurer MA, Laussen PC, Mayer JE Jr, Del Nido PJ, Pigula FA, et al. Factors associated with prolonged recovery after the fontan operation. Circulation. 2008;118(14 Suppl):S171-6.
9. Imura H, Caputo M, Lim K, Ochi M, Suleiman MS, Shimizu K, et al. Pulmonary injury after cardiopulmonary bypass: beneficial effects of low-frequency mechanical ventilation. $J$ Thorac Cardiovasc Surg. 2009;137:1530-7.

10. Portman MA, Slee A, Olson AK, Cohen G, Karl T, Tong E, et al. TRiiodothyronine supplementation in Infants and Children undergoing Cardiopulmonary bypass (TRICC): a multicenter placebo-controlled randomized trial: age analysis. Circulation. 2010;122(11 Suppl):S224-33.

11. Kaptein EM, Sanchez A, Beale E, Chan LS. Thyroid hormone therapy for postoperative nonthyroidal illnesses: a systematic review and synthesis. J Clin Endocrinol Metab. 2010;95:4526-34.

12. Pingitore A, Galli E, Barison A, Iervasi A, Scarlattini M, Nucci D, et al. Acute effects of triiodothyronine (T3) replacement therapy in patients with chronic heart failure and low-T3 syndrome: a randomized, placebo-controlled study. J Clin Endocrinol Metab. 2008;93:1351-8.

13. Ballard B, Torres LM, Romani A. Effect of thyroid hormone on $\mathrm{Mg}(2+)$ homeostasis and extrusion in cardiac cells. Mol Cell Biochem. 2008;318:117-27.

14. Belakavadi M, Saunders J, Weisleder N, Raghava PS, Fondell JD. Repression of cardiac phospholamban gene expression is mediated by thyroid hormone receptor- $\alpha 1$ and involves targeted covalent histone modifications. Endocrinology. 2010;151:2946-56

15. James SR, Ranasinghe AM, Venkateswaran R, McCabe CJ, Franklyn JA, Bonser RS. The effects of acute triiodothyronine therapy on myocardial gene expression in brain stem dead cardiac donors. J Clin Endocrinol Metab. 2010;95: 1338-43.

16. McMahon CK, Klein I, Ojamaa K. Interleukin-6 and thyroid hormone metabolism in pediatric cardiac surgery patients. Thyroid. 2003;13:301-4.

17. Hashimoto H, Igarashi N, Yachie A, Miyawaki T, Hashimoto T, Sato T. The relationship between serum levels of interleukin- 6 and thyroid hormone during the follow-up study in children with nonthyroidal illness: marked inverse correlation in Kawasaki and infectious disease. Endocr J. 1996;43:31-8.

18. Torpy DJ, Tsigos C, Lotsikas AJ, Defensor R, Chrousos GP, Papanicolaou DA. Acute and delayed effects of a single-dose injection of interleukin-6 on thyroid function in healthy humans. Metabolism. 1998;47:1289-93.

19. Sprague AH, Khalil RA. Inflammatory cytokines in vascular dysfunction and vascular disease. Biochem Pharmacol. 2009;78:539-52.

20. Hovels-Gurich HH, Schumacher K, Vazquez-Jimenez JF, Qing M, Huffmeier U, Buding B, et al. Cytokine balance in infants undergoing cardiac operation. Ann Thorac Surg. 2002;73:601-9.

21. Goser S, Ottl R, Brodner A, Dengler TJ, Torzewski J, Egashira K, et al. Critica role for monocyte chemoattractant protein-1 and macrophage inflammatory protein-1alpha in induction of experimental autoimmune myocarditis and effective anti-monocyte chemoattractant protein-1 gene therapy. Circulation. 2005; 112:3400-7.

22. Wassen FW, Moerings EP, Van Toor H, De Vrey EA, Hennemann G Everts ME. Effects of interleukin-1 beta on thyrotropin secretion and thyroid hormone uptake in cultured rat anterior pituitary cells. Endocrinology. 1996; 137:1591-8.

23. Allan CK, Newburger JW, McGrath E, Elder J, Psoinos C, Laussen PC, et al. The relationship between inflammatory activation and clinical outcome after infant cardiopulmonary bypass. Anesth Analg. 2010;111:1244-51. 


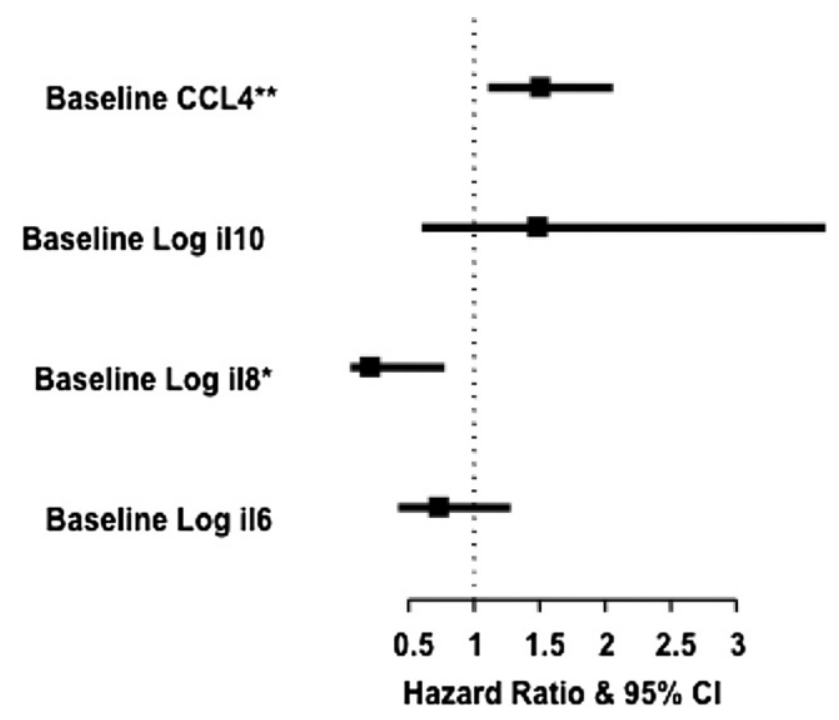

FIGURE E1. Baseline cytokine values are associated with differences in the chance of early extubation in untreated patients younger than 5 months old. $* P<.05 ; * * P<.01$. $C I$, Confidence interval; $C C L 4$, chemokine (C-C motif) ligand 4.

TABLE E1. Baseline characteristics for entire cohort versus cytokine analysis subset

\begin{tabular}{llcc}
\hline \multicolumn{1}{c}{ Characteristic } & \multicolumn{2}{c}{ Cytokine data } & $\boldsymbol{P}$ \\
\cline { 2 - 3 } & No $(\mathbf{n}=\mathbf{1 1 7})$ & Yes $(\mathbf{n}=\mathbf{7 6})$ & value \\
\hline Male gender (n) & $54(46 \%)$ & $30(39 \%)$ & .44 \\
Risk stratification & & & $.05^{*}$ \\
$\quad$ High & 31 & 31 & \\
$\quad$ Low & 86 & 45 & \\
Age (mo) & & & $.04 *$ \\
$\quad$ Median & 5.8 & 3.6 & \\
$\quad$ Range & $0-21.7$ & $0-23.2$ & \\
Baseline triiodothyronine (U) & 151 & 180 & $<.01 *$ \\
Mean intubation time (h) & 89 & 104 & .56 \\
Mean crossclamp time (min) & 62 & 62 & .98 \\
Mean bypass time (min) & 99 & 97 & .69 \\
Deaths (n) & 4 & 4 & .79 \\
\hline All $P$ values presented were derived from Student $t$ test, except for gender, risk, and \\
death, which were derived from a simple chi-square test. *Statistical significance \\
$(P \leq .05)$.
\end{tabular}

TABLE E2. Change in cytokine levels in untreated patients after cardiopulmonary bypass at 6 and 24 hours after crossclamping

\begin{tabular}{|c|c|c|c|c|c|c|c|c|}
\hline \multirow[b]{2}{*}{ Variable } & \multicolumn{4}{|c|}{ Change from baseline to $6 \mathrm{~h}$} & \multicolumn{4}{|c|}{ Change from baseline to $24 \mathrm{~h}$} \\
\hline & Patients (n) & Mean & $\mathbf{9 5} \% \mathrm{CI}$ & $P$ value & Patients (n) & Mean & $95 \% \mathrm{CI}$ & $P$ value \\
\hline IL-6 & 34 & 2.80 & 3.23 to 2.37 & $<.0001^{*}$ & 34 & 2.83 & 3.21 to 2.45 & $<.0001^{*}$ \\
\hline IL-8 & 37 & 1.11 & 1.42 to 0.81 & $<.0001^{*}$ & 37 & 1.09 & 1.39 to 0.78 & $<.0001 *$ \\
\hline IL-10 & 32 & 2.28 & 2.85 to 1.72 & $<.0001^{*}$ & 32 & 0.84 & 1.26 to 0.41 & .0003 \\
\hline IL-1 $\beta$ & 33 & 0.01 & 0.13 to -0.11 & .8923 & 33 & -0.01 & 0.09 to -0.11 & .8586 \\
\hline MCP1 & 38 & -0.17 & 0.03 to -0.38 & .0956 & 37 & -0.12 & 0.09 to -0.32 & .2562 \\
\hline MIP $1 \beta$ & 38 & -0.18 & 0.01 to -0.36 & .0602 & 38 & -0.48 & -0.30 to -0.66 & $<.0001 *$ \\
\hline
\end{tabular}

$C I$, Confidence interval; $I L$, interleukin; $M C P$, monocyte chemotactic protein; $M I P$, macrophage inflammatory protein. *Statistical significance $(P \leq .05)$.

943.e1 The Journal of Thoracic and Cardiovascular Surgery • October 2012 
TABLE E3. Six-hour cytokine levels associated with differences in triiodothyronine levels through 72 hours in untreated patients

\begin{tabular}{|c|c|c|c|c|c|c|}
\hline Variable & DF & Parameter estimate & SE & $t$ value & $\operatorname{Pr}>|\mathbf{t}|$ & $95 \% \mathrm{CI}$ \\
\hline 6-h R-square & & 0.4441 & & & & \\
\hline Intercept & 1 & 5.30402 & 0.16089 & 32.97 & $<.0001$ & 4.97496 to 5.63308 \\
\hline IL-10_6 & 1 & -0.06695 & 0.03475 & -1.93 & .0639 & -0.13802 to 0.00412 \\
\hline High risk & 1 & -0.28301 & 0.08473 & -3.34 & .0023 & -0.45630 to -0.10972 \\
\hline IL- $1 \beta-6$ & 1 & -0.39099 & 0.13190 & -2.96 & .0060 & -0.66075 to -0.12123 \\
\hline 12-h R-square & & 0.3522 & & & & \\
\hline Intercept & 1 & 5.20233 & 0.17535 & 29.67 & $<.0001$ & 4.84370 to 5.56097 \\
\hline IL-10_6 & 1 & -0.08030 & 0.03787 & -2.12 & .0427 & -0.15775 to -0.00284 \\
\hline High risk & 1 & -0.15740 & 0.09235 & -1.70 & .0990 & -0.34627 to 0.03146 \\
\hline IL- $1 \beta-6$ & 1 & -0.46652 & 0.14375 & -3.25 & .0030 & -0.76052 to -0.17252 \\
\hline 24-h R-square & & 0.3229 & & & & \\
\hline Intercept & 1 & 4.94740 & 0.17787 & 27.81 & $<.0001$ & 4.58361 to 5.31119 \\
\hline IL-10_6 & 1 & -0.10474 & 0.03842 & -2.73 & .0107 & -0.18331 to -0.02617 \\
\hline High risk & 1 & -0.01538 & 0.09367 & -0.16 & .8707 & -0.20696 to 0.17620 \\
\hline IL- $1 \beta-6$ & 1 & -0.46901 & 0.14582 & -3.22 & .0032 & -0.76724 to -0.17079 \\
\hline 72-h R-square & & 0.1996 & & & & \\
\hline Intercept & 1 & 5.12113 & 0.37185 & 13.77 & $<.0001$ & 4.34997 to 5.89230 \\
\hline IL-10_6 & 1 & -0.08782 & 0.07566 & -1.16 & .2582 & -0.24474 to 0.06909 \\
\hline High risk & 1 & -0.08639 & 0.18143 & -0.48 & .6387 & -0.46264 to 0.28987 \\
\hline IL- $1 \beta-6$ & 1 & -0.65791 & 0.28984 & -2.27 & .0334 & -1.25901 to -0.05682 \\
\hline
\end{tabular}

$C I$, Confidence interval; $D F$, degrees of freedom; $I L$, interleukin; $S E$, standard error.

TABLE E4. Anatomic diagnoses

Isolated ventricular septal defect

Tetralogy of Fallot

Transposition of the great arteries

Hypoplastic left ventricle

Complete atrioventricular canal defect

Total anomalous pulmonary venous drainage

Other low-risk cardiopulmonary bypass

Other high-risk cardiopulmonary bypass

Superior vena cava to pulmonary anastomosis 